\title{
PAQR3 inhibits proliferation via suppressing PI3K/AKT signaling pathway in non-small cell lung cancer
}

Xiaohui Li, Mengfei Li, Dong Chen, Gongning Shi, Hui Zhao

Department of Cardiothoracic Surgery, Huaihe Hospital of Henan University, Kaifeng, China

Submitted: 10 October 2017

Accepted: 29 October 2017

Arch Med Sci 2018; 14, 6: 1289-1297

DOI: https://doi.org/10.5114/aoms.2017.72220

Copyright $\odot 2017$ Termedia \& Banach

\section{Abstract}

Introduction: Lung cancer is the leading cause of cancer-related death worldwide and non-small cell lung cancer (NSCLC) accounts for approximately $85 \%$ of all lung cancer cases. PAQR (progestin and adipoQ receptor family) 3, a Golgi-anchored membrane protein, has been demonstrated to act as a tumor suppressor in multiple cancers. However, the expression and role of PAQR3 have never been explored in NSCLC. The purpose of this study was to investigate the expression and role of PAQR3 in NSCLC.

Material and methods: Expression of PAQR3 at mRNA and protein levels was determined by qRT-PCR and western blot, respectively. Cell proliferation was analyzed by MTT assay. Apoptosis and cell cycle distribution were evaluated by flow cytometry.

Results: The expression of PAQR3 was downregulated in NSCLC tissue samples and cell lines at both mRNA and protein levels $(p<0.05)$. Overexpression of PAQR3 significantly inhibited cell proliferation, induced apoptosis and promoted cell cycle arrest at GO/G1 phase in NSCLC cell lines $(p<0.05)$. In contrast, knockdown of PAQR3 showed a reverse effect on NSCLC cells $(p<0.05)$. Moreover, PAQR3 may exert its tumor suppressive roles via suppressing the PI3K/AKT signaling pathway in NSCLC.

Conclusions: Our findings suggest that PAQR3 is a tumor suppressor in the development of NSCLC and may serve as a novel therapeutic target in the treatment of patients with NSCLC.

Key words: NSCLC, PAQR3, proliferation, PI3K/AKT.

\section{Introduction}

Lung cancer is one of the most prevalent malignancies and the leading cause of cancer-related death in adults worldwide, with an estimated 1.4 million deaths per year [1]. Non-small cell lung cancer (NSCLC) represents the majority of lung cancers and accounts for approximately $85 \%$ of all lung cancer cases [2]. According to the histological features, NSCLC is mainly categorized into adenocarcinoma, squamous cell carcinoma and large cell carcinoma and others [3]. Despite the great progress in the combination of surgical resection, radiotherapy and chemotherapy in the treatment of NSCLC, the prognosis of NSCLC patients still remains poor and the 5-year overall survival rate is only $15 \%$ [4]. Therefore, greater understanding of its initiation and development may provide a novel approach for the diagnosis and treatment of NSCLC patients.

\author{
Corresponding author: \\ Dr. Dong Chen \\ Department of \\ Cardiothoracic Surgery \\ Huaihe Hospital \\ of Henan University \\ No. 8 Baobei Road \\ Gulou District \\ 475000 Kaifeng, China \\ Phone: +860371 23906599 \\ E-mail: doczhaoh@163.com
}


The progestin and adipoQ receptor family (named PAQR) is a highly conserved protein family composed of 11 members, PAQR1 to PAQR11 [5]. PAQR3, also known as Raf kinase trapping to Golgi (RKTG), is a Golgi-anchored membrane protein containing seven transmembrane helices and interacts with the $G \beta$ subunit via the first 20 amino acids of its $\mathrm{N}$-terminus and retains $\mathrm{G} \beta \gamma$ at the Golgi $[6,7]$. An increasing number of studies have shown that PAQR3 functions as a tumor suppressor by regulating tumor cell proliferation and migration in various human cancers such as glioma [8], gastric cancer [9] and prostate cancer [10]. Previous studies have reported that PAQR3 exerts its tumor suppressive roles by inhibiting the Ras/Raf/MEK and PI3K/AKT signaling pathways [11-13]. However, the role of PAQR3 in the development of NSCLC has never been reported.

In the present study, we investigated the expression pattern of PAQR3 and its potential functions in NSCLC. Our study revealed that PAQR3 is significantly downregulated in NSCLC tissues and cell lines when compared with the control. Overexpression of PAQR3 significantly inhibited cell proliferation while knockdown of PAQR3 promoted cell proliferation in NSCLC. Moreover, PAQR3 was shown to inhibit cell proliferation via suppressing the PI3K/AKT signaling pathway in NSCLC. Our findings suggest that PAQR3 is a tumor suppressor in NSCLC and may serve as a therapeutic target in the treatment of patients with NSCLC.

\section{Material and methods}

\section{Tissue samples}

A total of 20 paired NSCLC specimens and matched adjacent normal tissues were collected from patients with primary NSCLC who had undergone surgical treatment at our department. All tissues were frozen in liquid nitrogen immediately until use. The diagnoses were confirmed by pathological analysis. Written informed consent was obtained from each patient and our study was approved by the Ethics Committees of Huaihe Hospital of Henan University. This study was performed following the Declaration of Helsinki.

\section{Cell lines}

Human NSCLC cell lines (SK-MES-1, A549, SPCA-1 and H1229) and the non-tumorous human bronchial epithelium cell line BEAS-2B were all obtained from the Cell Bank, China Academy of Sciences (Shanghai, China). All cells were maintained in RPMI 1640 medium supplemented with $10 \%$ fetal bovine serum (both Gibco; Thermo Fisher Scientific, Inc., Waltham, MA, USA), $100 \mathrm{U} / \mathrm{ml}$ of penicillin and $100 \mu \mathrm{g} / \mathrm{ml}$ of streptomycin. Cells were maintained in a humidified incubator containing $5 \% \mathrm{CO} 2$ at $37^{\circ} \mathrm{C}$.

\section{RNA extraction and qRT-PCR}

Total RNA was extracted from tissue specimens or cultured cells using TRIzol (Invitrogen) according to the manufacturer's instruction. cDNA was synthesized from total RNA by using the PrimeScript RT Reagent Kit (TaKaRa, Dalian, China). Quantitative real-time PCR (qRT-PCR) was performed using SYBR Green PCR Master Mix (Applied Biosystems, Foster (ity, USA) in an Applied Biosystems 7500 sequence detection system (Applied Biosystems) following the manufacturer's guidelines. The quantitative real-time PCR conditions were: $95^{\circ} \mathrm{C}$ for $10 \mathrm{~min}, 40 \mathrm{cycles}$ of $95^{\circ} \mathrm{C}$ for $15 \mathrm{~s}$, and $60^{\circ} \mathrm{C} 1 \mathrm{~min}$. The relative expression level of PAQR3 was calculated by using the $2^{-\Delta \Delta c t}$ method and GAPDH was used as an internal control. The primers for PAQR3 were 5'-AACCCGTACATCACCGACG-3' (forward) and 5'-TCTGGACGCACTTGCTGAAG-3' (reverse) The primers for GAPDH were $5^{\prime}$-CATCTTCTTTTGCGTCGCCA-3' (forward) and 5'-TTAAAAGCAGCCCTGGTGACC-3' (reverse). Each experiment was independently repeated three times.

\section{Western blot}

The total protein was extracted from tissues or cultured cells by RIPA buffer and quantified by the bicinchoninic acid (BCA) kit (Pierce, Rockford, IL, USA). An equal amount of protein was isolated by $10 \%$ SDS-PAGE and transferred to nitrocellulose membranes (Amersham BioSciences, Buckinghamshire, UK). The membranes were blocked with $5 \%$ non-fat milk in PBS for $1 \mathrm{~h}$ and incubated with primary antibodies at $4^{\circ} \mathrm{C}$ overnight. After washing three times, membranes were immunoblotted by secondary antibodies (1 : 15000, LI-COR, Nebraska, USA) for $1 \mathrm{~h}$ at room temperature and an infrared imaging system (LI-COR Biosciences, Lincoln, USA) was used to visualize the protein bands. The primary antibodies against GAPDH and PAQR3 were purchased from Santa Cruz Biotechnology (Santa Cruz, CA, USA). Other primary antibodies were all purchased from Cell Signaling Technology (CST, Boston, USA).

\section{Cell transfection}

The transfections were all performed using Lipofectamine 2000 (Invitrogen) according to the manufacturer's instructions. For PAQR3 overexpression, PAQR3 cDNA was purified and cloned into a pcDNA3.1 vector (Promega, Madison, WI, USA) to construct the pcDNA3.1-PAQR3 recombinant plasmids (PAQR3). The empty vector was used as the control (vector). For PAQR3 downregulation, siRNA targeting human PAQR3 (si-PAQR3) 
and the scramble oligonucleotide (si-NC) was synthesized by GenePharma (Shanghai, China). The sequences of si-NC were 5'-UUCUCCGAACGUGUCACGUTT-3' (sense) and 5'-ACGUGACACGUUCGGAGAATT-3' (antisense). The sequences of si-PAQR3 were 5'-GCCACCAAAUAUGGCAUAUTT-3' (sense) and 5'-AUAUGCCAUAUUUGGUGGCTT-3' (antisense). qRT-PCR and western blot were used to confirm the expression level of PAQR3 in treated cells.

\section{MTT assay}

Cell proliferation was measured by the 3-(4,5-dimethylthiazol-2-yl)-2, 5-diphenyl tetrazolium bromide (MTT) assay according to the manufacturer's protocol. In brief, cells were plated into a 96-well plate and cultured at $37^{\circ} \mathrm{C}$ for different time periods. Then, $20 \mu \mathrm{M}$ MTT ( $5 \mathrm{mg} / \mathrm{ml}$, Sigma) was added to each wells and incubated at $37^{\circ} \mathrm{C}$ for further $4 \mathrm{~h}$. Subsequently, $150 \mu \mathrm{l}$ of DMSO was added for $15 \mathrm{~min}$ to stop the reaction. The optical density at $570 \mathrm{~nm}$ was determined using a microtiter plate reader (Molecular Devices, Sunnyvale, CA, USA). All experiments were performed in triplicate.

\section{Flow cytometry}

Cell apoptosis was measured using flow cytometry with the FITC-Annexin V Apoptosis Detection Kit (BD Biosciences, CA, USA) following the manufacturer's protocol. Briefly, cells were harvested, washed twice with cold PBS and resuspended in $200 \mu \mathrm{l}$ of binding buffer. Then, the cells were treated with FITC Annexin $\mathrm{V}$ and propidium iodide solution in darkness at room temperature for $15 \mathrm{~min}$. The apoptosis rate was detected using a Calibur Flow Cytometer (Becton, Dickinson and Company, CA, USA). For cell cycle analysis, cells were collected, washed twice with cold PBS and fixed with $70 \%$ ethanol overnight at $4^{\circ} \mathrm{C}$. Subsequently, cells were washed twice and stained with propidium iodide (PI, Sigma) for $30 \mathrm{~min}$ at room temperature. Cells in each phase were counted by a Calibur Flow Cytometer. Three independent experiments were performed.

A

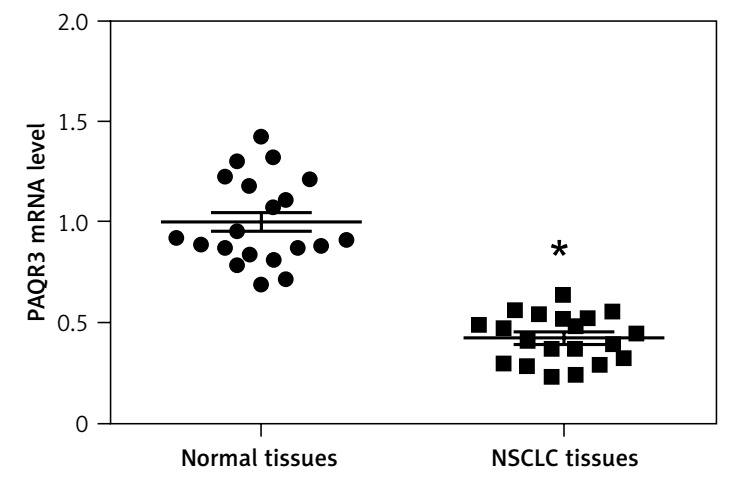

B

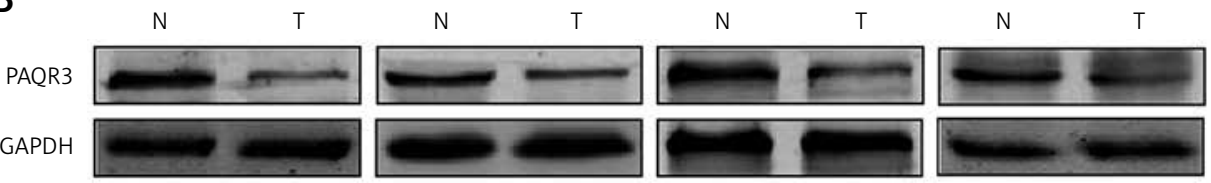

C

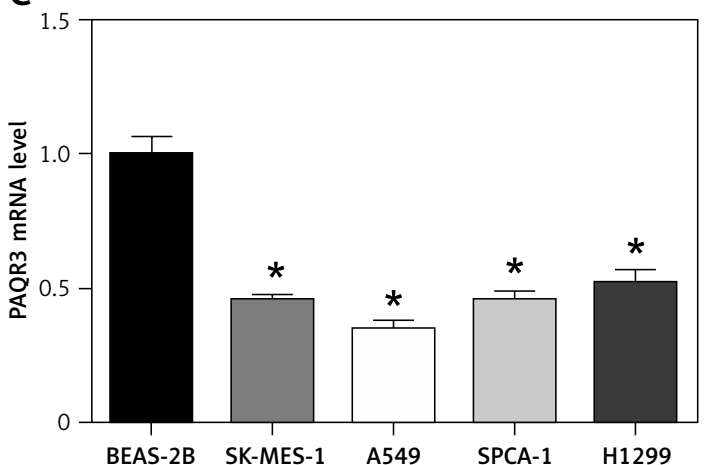

D

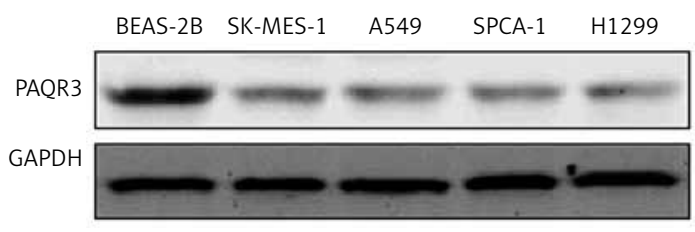

Figure 1. PAQR3 was down-regulated in NSCLC tissues and cell lines. A - PAQR3 mRNA level in CC tissues and the matched normal tissues as determined by qRT-PCR. B - Representative images of the PAQR3 protein expression in NSCLC tissues and the matched normal tissues as detected by western blot. C - PAQR3 mRNA level in NSCLC cell lines (SK-MES-1, A549, SPCA-1 and H1229) and the non-tumorous human bronchial epithelium cell line BEAS-2B as determined by qRT-PCR. D - PAQR3 protein expression in NSCLC cell lines and BEAS-2B cells detected by western blot Data are expressed as mean $\pm S D$. ${ }^{*} P<0.05$. 

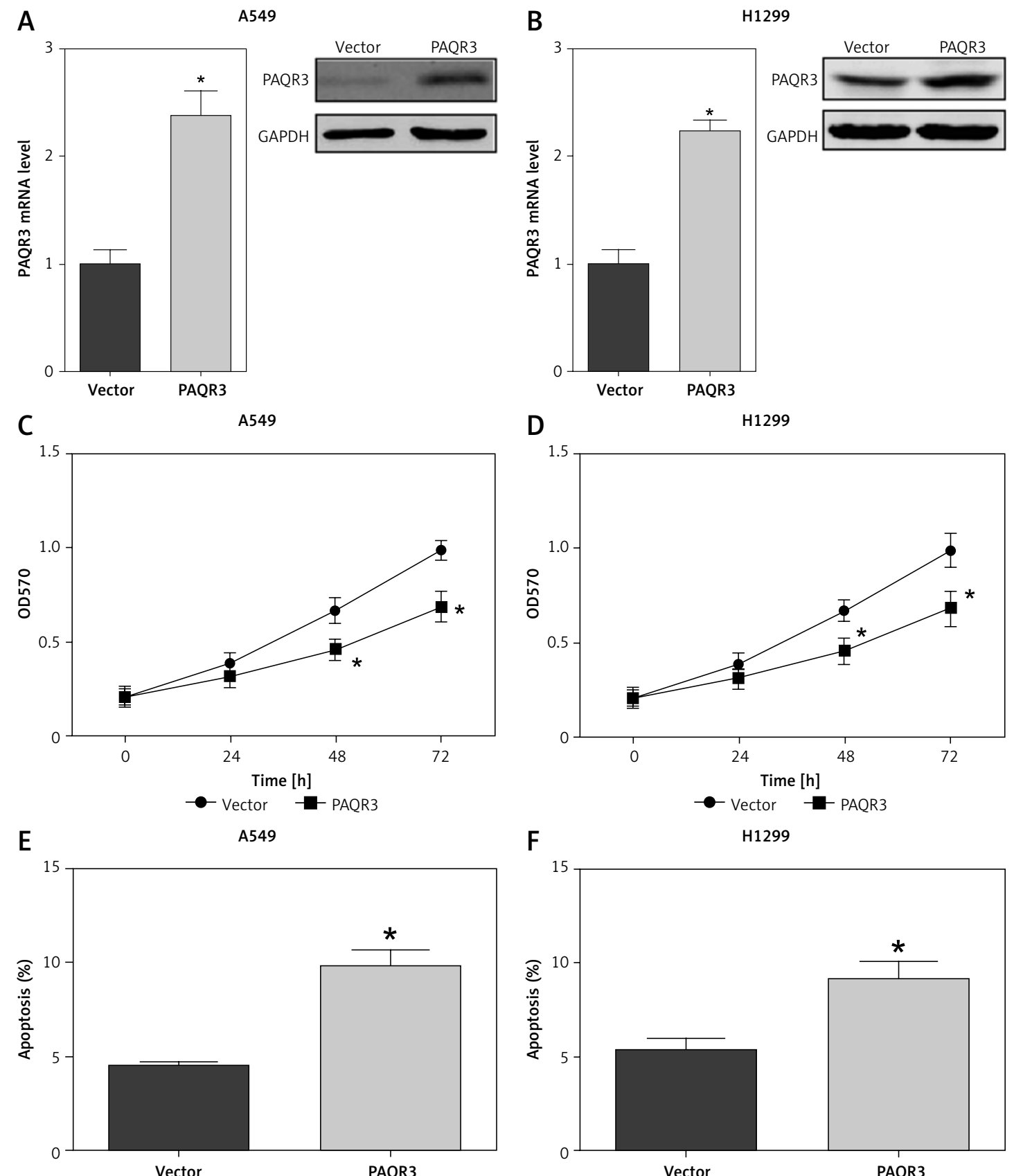

$\mathbf{F}$

H1299

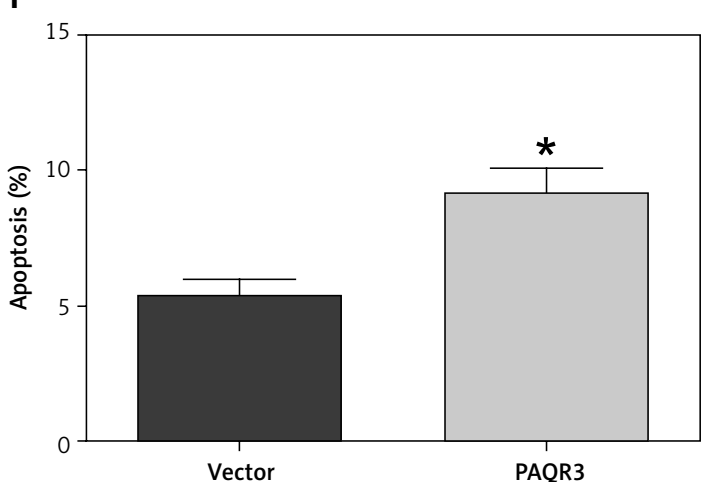

Figure 2. Overexpression of PAQR3 inhibited NSCLC cell proliferation. A, B - mRNA and protein levels of PAQR3 in NSCLC cell lines A549 and H1299 following pcDNA3.1-PAQR3 plasmid or empty vector transfection. C, D - Cell proliferation of NSCLC cells as analyzed by MTT assay after PAQR3 overexpression. E, F-Cell apoptosis of NSCLC cells as measured by flow cytometry after PAQR3 overexpression

Data are expressed as mean $\pm S D$. ${ }^{*} P<0.05$. 
G
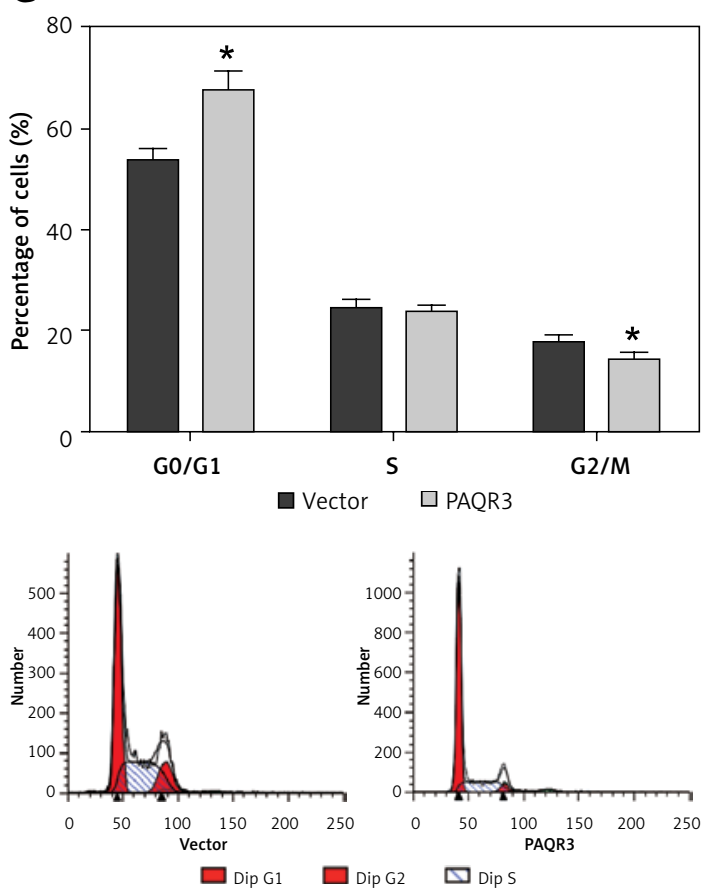

$\mathrm{H}$
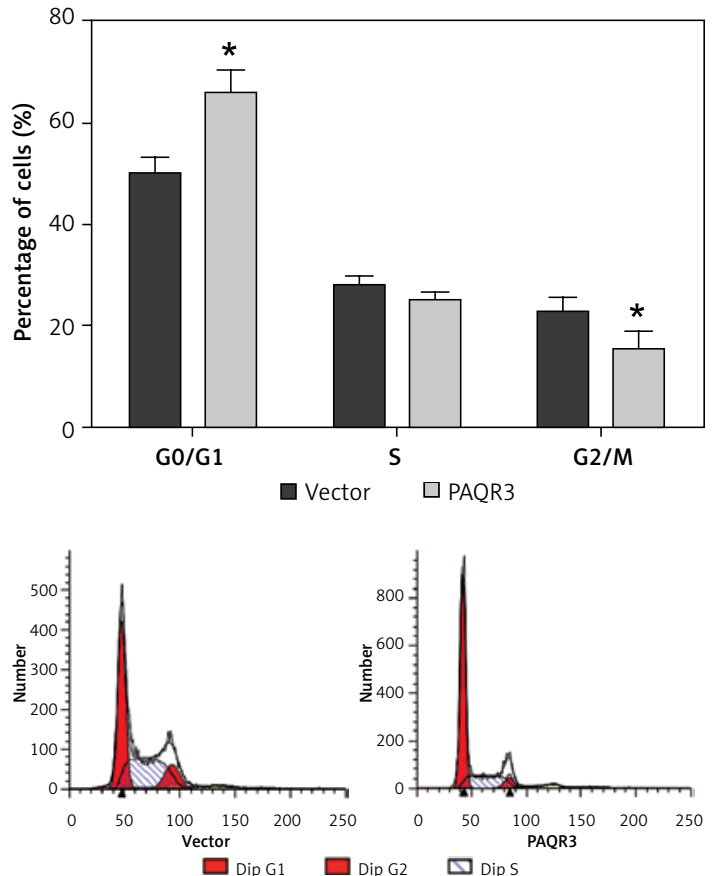

Figure 2. Cont. G, H - Cell cycle distribution of NSCLC cells as assessed by flow cytometry after PAQR3 overexpression Data are expressed as mean $\pm S D$. ${ }^{*} P<0.05$.

\section{Statistical analysis}

All data were expressed as mean \pm SD. Differences between groups were compared by Student's $t$-test or one-way analysis of variance using SPSS 17.0 software (SPSS, Inc., Chicago, IL, USA). Differences were considered to be statistically significant when $p<0.05$.

\section{Results}

\section{PAQR3 was down-regulated in NSCLC} tissues and cell lines

To elucidate the potential roles of PAQR3 in the development of NSCLC, we detected its expression patterns at both mRNA and protein levels in NSCLC tissues and cell lines. We found that the mRNA and protein levels of PAQR3 were both down-regulated in NSCLC tissues as determined by qRT-PCR (Figure $1 \mathrm{~A}$ ) and western blot (Figure $1 \mathrm{~B}$ ), respectively. In addition, PAQR3 was also found to be down-regulated in NSCLC cell lines at both mRNA and protein levels (Figures 1 C, D). These results suggested that PAQR3 may act as a tumor suppressor in NSCLC.

\section{Overexpression of PAQR3 inhibited NSCLC cell proliferation}

To examine the biological functions of PAQR3 in NSCLC, PAQR3 was overexpressed in NSCLC cell lines A549 and H1299 by pcDNA3.1-PAQR3 recombinant plasmid. The PAQR3 expression level was significantly increased in A549 and H1299 cells following plasmid transfection (Figures $2 \mathrm{~A}, \mathrm{~B})$. Then, the MTT assay was conducted to evaluate cell proliferation and the results showed that overexpression of PAQR3 significantly inhibited NSCLC cell proliferation (Figures 2 C, D). As cell proliferation is always correlated with apoptosis and cell cycle distribution, we analyzed NSCLC cell apoptosis and cell cycle distribution after PAQR3 overexpression. The data showed that PAQR3 overexpression significantly induced apoptosis (Figures $2 \mathrm{E}, \mathrm{F}$ ) and resulted in cell cycle arrest at G0/G1 phase (Figures $2 \mathrm{G}, \mathrm{H}$ ) in NSCLC cell lines.

\section{Knockdown of PAQR3 promoted NSCLC cell proliferation}

To further clarify the tumor suppressive roles of PAQR3 in NSCLC, the expression of PAQR3 was knocked down in NSCLC cell lines A549 and H1299. The knockdown efficiency was confirmed by qRTPCR and western blot (Figures $3 A, B$ ). Functional assays showed that knockdown of PAQR3 markedly promoted NSCLC cell proliferation (Figures 3 C, D). In addition, the cell apoptosis rate (Figures $3 \mathrm{E}, \mathrm{F}$ ) and cell amount at GO/G1 phase (Figures $3 \mathrm{G}, \mathrm{H}$ ) of NSCLC cells were obviously decreased in the PAQR3 knockdown group in comparison with the control group. 

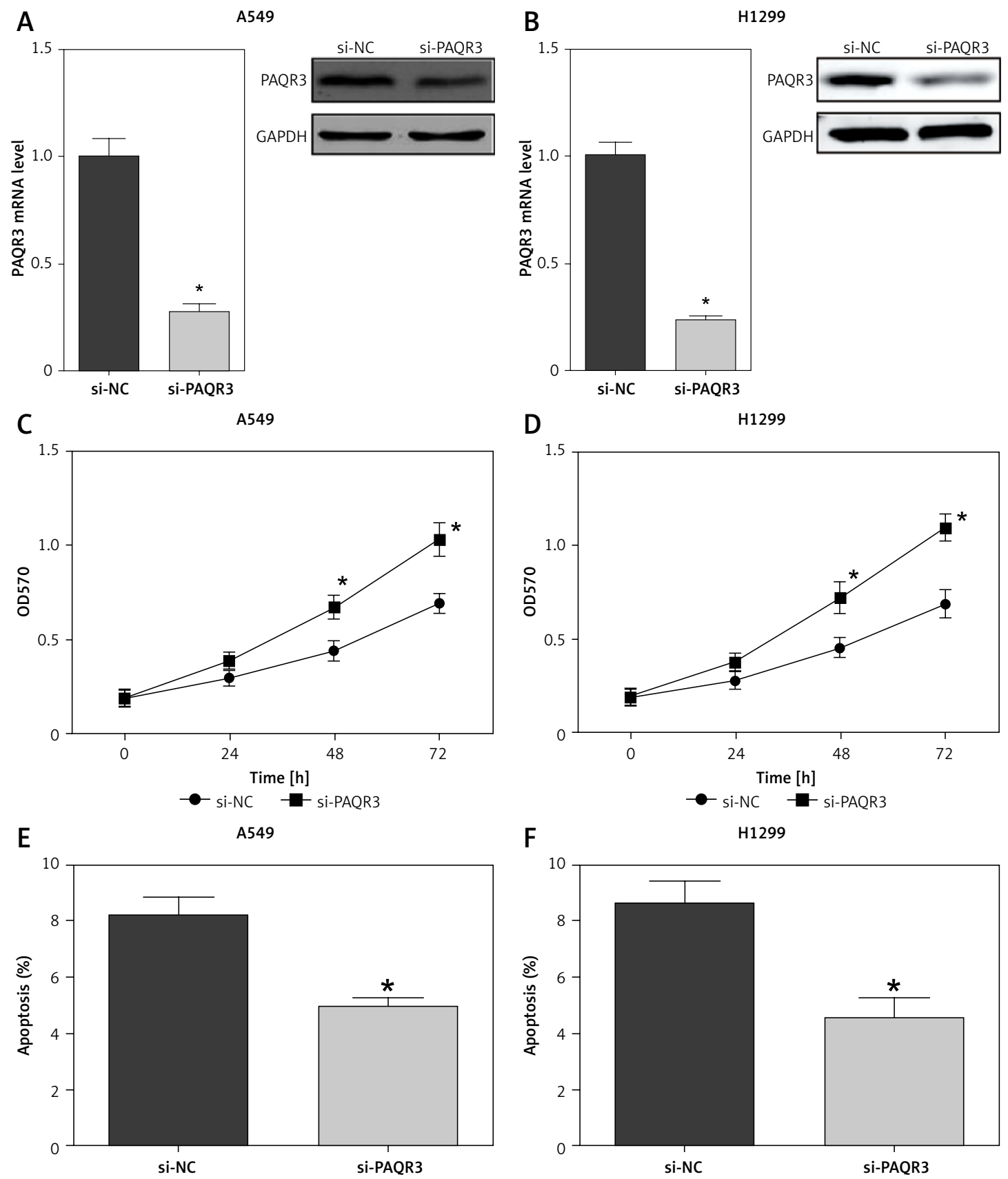

F

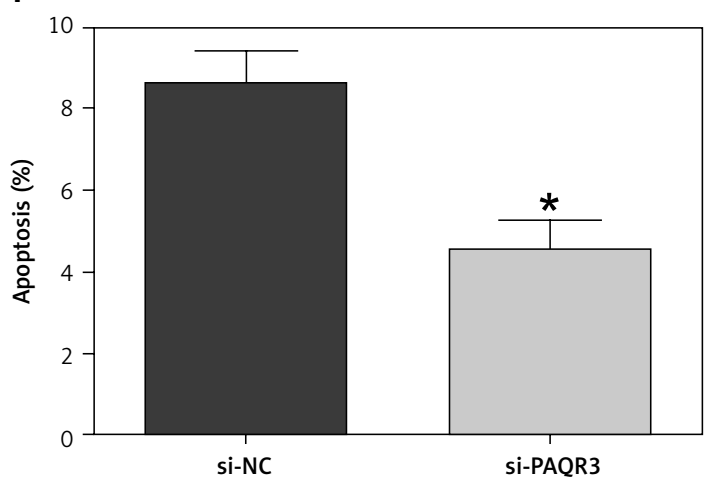

Figure 3. Knockdown of PAQR3 promoted NSCLC cell proliferation. A, B - mRNA and protein levels of PAQR3 in NSCLC cell lines A549 and H1299 following PAQR3 knockdown. C, D - Cell proliferation of NSCLC cells as analyzed by MTT assay after PAQR3 knockdown. E, F - Cell apoptosis of NSCLC cells as measured by flow cytometry after PAQR3 knockdown

Data are expressed as mean $\pm S D .{ }^{*} P<0.05$. 
G
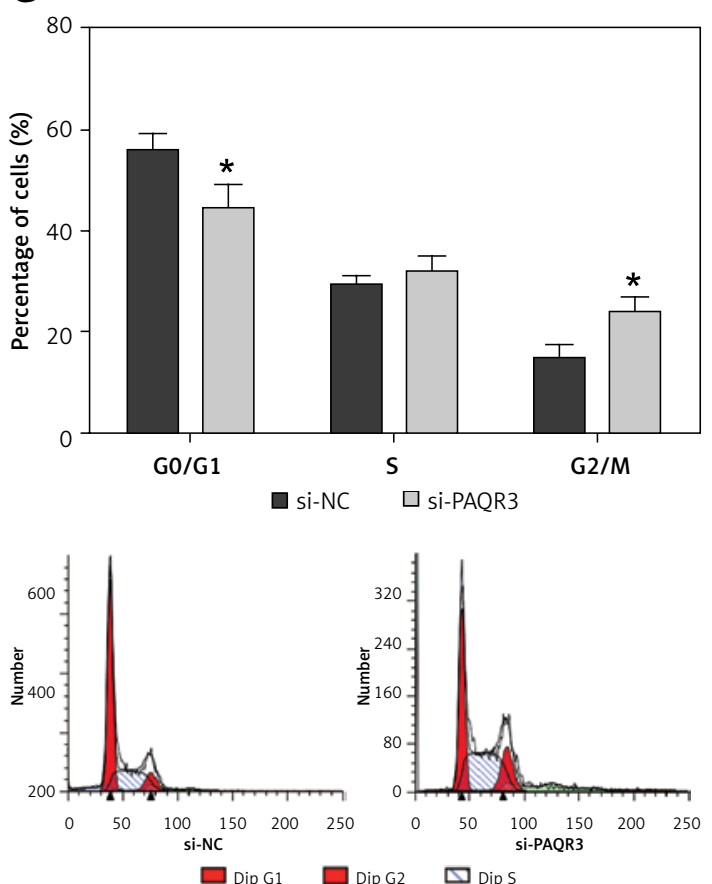

$\mathrm{H}$
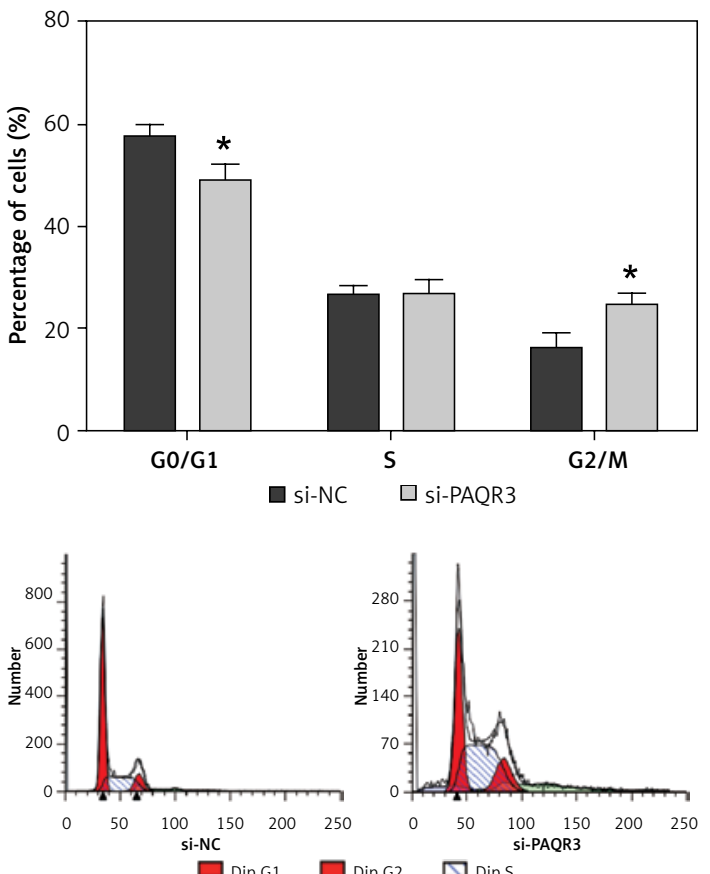

Figure 3. Cont. G, H - Cell cycle distribution of NSCLC cells as assessed by flow cytometry after PAQR3 knockdown Data are expressed as mean $\pm S D$. ${ }^{*} P<0.05$.

PAQR3 may exert its tumor suppressive roles via suppressing PI3K/AKT signaling pathway in NSCLC

As the PI3K/AKT pathway is crucial for cell proliferation in the development of human cancers, we supposed that PAQR3 may also affect the PI3K/ AKT pathway in NSCLC and verified that using western blot. We found that the phosphorylation of PI3K and AKT was significantly downregulated in NSCLC cells A549 and H1299 following PAQR3 overexpression (Figure 4). These results suggested that PAQR3 may exert its tumor suppressive roles in NSCLC via suppressing the PI3K/AKT signaling pathway.

\section{Discussion}

PAQR3 was first identified as a spatial regulator of Raf kinase and was named RKTG. It binds Raf-1, translocates Raf-1 into the Golgi apparatus, inhibits Raf- 1 activation, reduces the association of Raf- 1 with Ras and MEK, and blocks the ERK pathway [11]. Since then, an increasing number of studies have shown that PAQR3 acts as a tumor suppressor in multiple human cancers. For example, Zhang et al. reported that PAQR3 inhibits cell proliferation, migration, sprouting and angiogenesis by suppressing MAPK-mediated autocrine VEGF signaling in clear cell renal cell carcinoma [14]. Wu et al. showed that PAQR3 is downregulated in hepatocellular carcinoma and the decreased expres- sion of PAQR3 is correlated with a poor prognosis [15]. Ma et al. found that PAQR3 is decreased in osteosarcoma and overexpression of PAQR3 inhibited osteosarcoma cell proliferation, migration, and invasion by promoting ERK phosphorylation [16]. In addition, PAQR3 was also demonstrated to suppress the tumorigenesis of colorectal cancers [17] and skin carcinogenesis [18] in a mouse model. These studies indicated the tumor suppressive effect of PAQR3 in cancer development.

In the current study, we demonstrated for the first time that the expression pattern of PAQR3 was down-regulated in NSCLC tissues and cell lines at both mRNA and protein levels. Therefore, we supposed that PAQR3 may function as a tumor suppressor in NSCLC and conducted a series of in vitro functional experiments. Our results showed that overexpression of PAQR3 significantly inhibited cell proliferation, induced apoptosis and promoted cell cycle arrest at G0/G1 phase in NSCLC cells. However, knockdown of PAQR3 promoted NSCLC cell proliferation, suppressed apoptosis and increased the cell amount at G0/G1 phase. Moreover, mechanistic studies showed that phosphorylation of PI3K and AKT was downregulated following PAQR3 overexpression in NSCLC cells.

The PI3K/AKT signaling pathway is one of the most commonly activated signaling pathways in various types of tumors including NSCLC [19-21]. Drugs targeting the PI3K/AKT pathway have great potential to exert their anticancer effects [22]. 
A

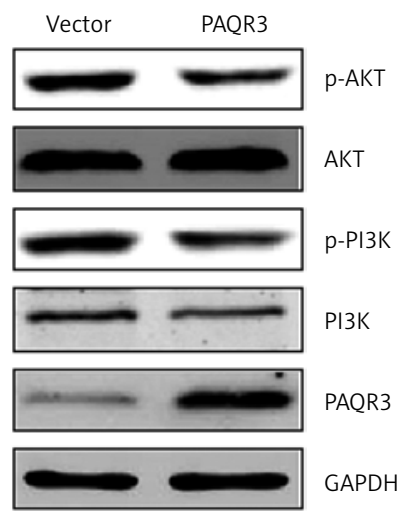

A549

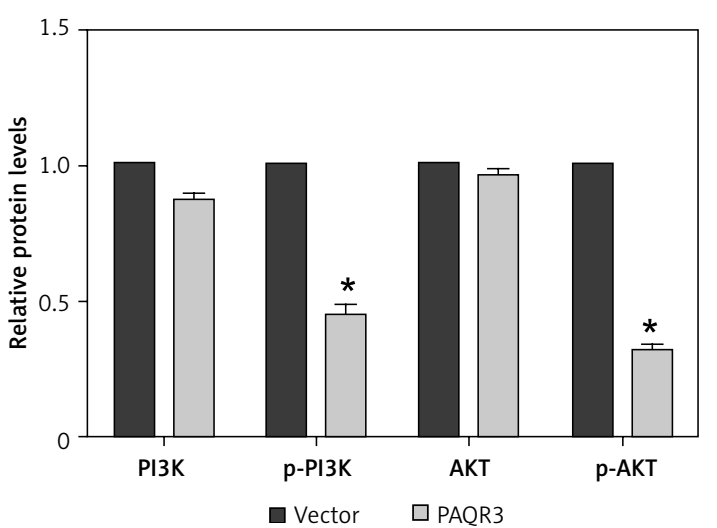

B

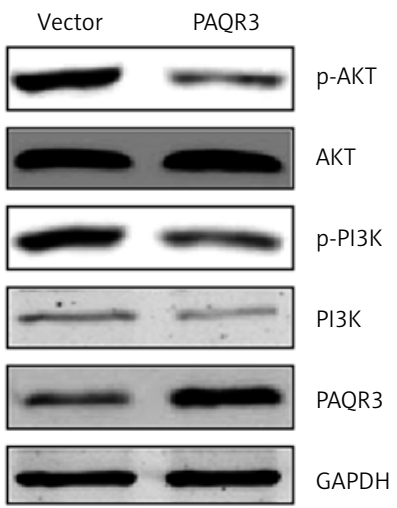

H1299

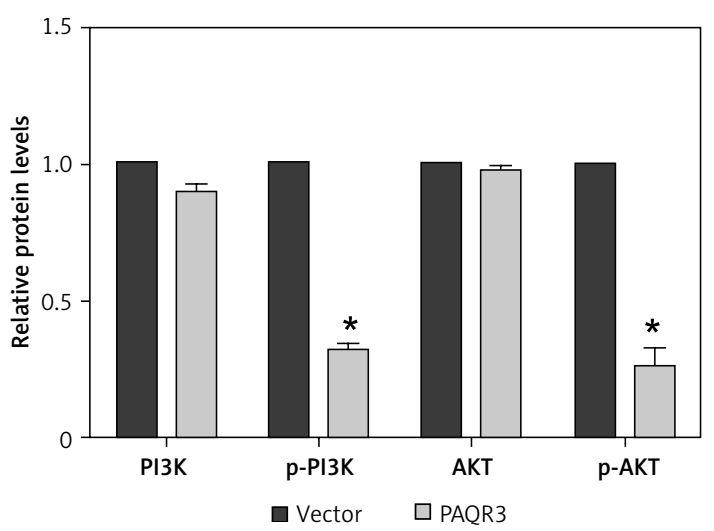

Figure 4. PAQR3 may exert its tumor suppressive roles via suppressing PI3K/AKT signaling pathway in NSCLC. A - Protein expression of PI3K and AKT and phosphorylated PI3K and AKT in A549 cells following PAQR3 overexpression. Relative quantities are shown. B - Protein expression of PI3K and AKT and phosphorylated PI3K and AKT in $\mathrm{H} 1299$ cells following PAQR3 overexpression. Relative quantities are shown

Therapeutic methods targeting PI3K/AKT are widely used in the treatment of human cancers such as prostate cancer [23], osteosarcoma [24] and neuroblastoma [25]. PAQR3 was also reported to exert its functions by regulating the PI3K/Akt signaling pathway $[8,13]$. Similarly, our study demonstrated that overexpression of PAQR3 suppressed the phosphorylation of PI3K and AKT in NSCLC cells.

In recent years, identifying novel molecules involved in the development of human cancers is important to obtain a full understanding of cancer initiation and helpful for the treatment of cancers. For instance, Zhao et al. showed that miR-630 functions as an oncogene in renal cell carcinoma and may act as a potential therapeutic target for renal cancer treatment [26]. Zhang et al. found that CtBP2 is overexpressed in prostate cancer and its overexpression is associated with tumorigenesis and poor clinical outcome of prostate cancer [27]. In the present study, we found that PAQR3 is involved in the development of NSCLC and may act as a therapeutic target in the treatment of NSCLC.

Overall, our study demonstrated that PAQR3 was down-regulated in NSCLC. PAQR3 functions as a tumor suppressor by inhibiting cell proliferation, inducing apoptosis and promoting cell cy- cle arrest via suppressing the PI3K/AKT signaling pathway in NSCLC. These findings suggest that PAQR3 may serve as a novel therapeutic target in NSCLC treatment.

\section{Acknowledgments}

Xiaohui Li and Mengfei Li are equal contributors.

\section{Conflict of interest}

The authors declare no conflict of interest.

\section{References}

1. Torre LA, Bray F, Siegel RL, Ferlay J, Lortet-Tieulent J, Jemal A. Global cancer statistics, 2012. CA Cancer J Clin 2015; 65: 87-108.

2. Subramaniam S, Thakur RK, Yadav VK, Nanda R, Chowdhury S, Agrawal A. Lung cancer biomarkers: state of the art. J Carcinog 2013; 12: 3.

3. Koudelakova V, Kneblova M, Trojanec R, Drabek J, Hajduch M. Non-small cell lung cancer: genetic predictors. Biomedical papers of the Medical Faculty of the University Palacky, Olomouc, Czechoslovakia 2013; 157: 125-36.

4. Ramnath N, Dilling TJ, Harris LJ, et al. Treatment of stage III non-small cell lung cancer: diagnosis and manage- 
ment of lung cancer, $3^{\text {rd }}$ ed: American College of Chest Physicians evidence-based clinical practice guidelines. Chest 2013; 143 (5 Suppl): e314S-40S.

5. Tang YT, Hu T, Arterburn M, et al. PAQR proteins: a novel membrane receptor family defined by an ancient 7 -transmembrane pass motif. J Mol Evol 2005; 61: 372-80.

6. Luo X, Feng L, Jiang X, et al. Characterization of the topology and functional domains of RKTG. Biochem $J$ 2008; 414: 399-406.

7. Jiang $Y$, Xie $X$, Zhang $Y$, et al. Regulation of G-protein signaling by RKTG via sequestration of the $G$ betagamma subunit to the Golgi apparatus. Mol Cell Biol 2010; 30: 78-90.

8. Tang SL, Gao YL, Hu WZ. PAQR3 inhibits the proliferation, migration and invasion in human glioma cells. Biomed Pharmacother 2017; 92: 24-32.

9. Guo W, You X, Xu D, et al. PAQR3 enhances Twist1 degradation to suppress epithelial-mesenchymal transition and metastasis of gastric cancer cells. Carcinogenesis 2016; 37: 397-407.

10. Huang W, Guo W, You X, et al. PAQR3 suppresses the proliferation, migration and tumorigenicity of human prostate cancer cells. Oncotarget 2016; 8: 53948-58.

11. Feng L, Xie X, Ding Q, et al. Spatial regulation of Raf kinase signaling by RKTG. Proc Natl Acad Sci USA 2007; 104: 14348-53.

12. Fan F, Feng L, He J, et al. RKTG sequesters B-Raf to the Golgi apparatus and inhibits the proliferation and tumorigenicity of human malignant melanoma cells. Carcinogenesis 2008; 29: 1157-63.

13. Wang $X$, Wang L, Zhu L, et al. PAQR3 modulates insulin signaling by shunting phosphoinositide 3-kinase p110alpha to the Golgi apparatus. Diabetes 2013; 62: 444-56.

14. Zhang Y, Jiang X, Qin X, et al. RKTG inhibits angiogenesis by suppressing MAPK-mediated autocrine VEGF signaling and is downregulated in clear-cell renal cell carcinoma. Oncogene 2010; 29: 5404-15.

15. Wu HG, Zhang WJ, Ding Q, et al. Identification of PAQR3 as a new candidate tumor suppressor in hepatocellular carcinoma. Oncol Rep 2014; 32: 2687-95.

16. Ma Z, Wang Y, Piao T, et al. The tumor suppressor role of PAQR3 in osteosarcoma. Tumour Biol 2015; 36: 3319-24.

17. Wang X, Li X, Fan F, et al. PAQR3 plays a suppressive role in the tumorigenesis of colorectal cancers. Carcinogen esis 2012; 33: 2228-35.

18. Xie $X$, Zhang $Y$, Jiang $Y$, et al. Suppressive function of RKTG on chemical carcinogen-induced skin carcinogenesis in mouse. Carcinogenesis 2008; 29: 1632-8.

19. Liu JY, Wu XY, Wu GN, Liu FK, Yao XQ. FOXQ1 promotes cancer metastasis by PI3K/AKT signaling regulation in colorectal carcinoma. Am J Transl Res 2017; 9: 2207-18.

20. Yin K, Wang L, Zhang $X$, et al. Netrin-1 promotes gastric cancer cell proliferation and invasion via the receptor neogenin through PI3K/AKT signaling pathway. Oncotarget 2017; 8: 51177-89.

21. Xia H, Li Y, Lv X. MicroRNA-107 inhibits tumor growth and metastasis by targeting the BDNF-mediated PI3K/ AKT pathway in human non-small lung cancer. Int J Oncol 2016; 49: 1325-33.

22. Mayer IA, Arteaga CL. The PI3K/AKT pathway as a target for cancer treatment. Ann Rev Med 2016; 67: 11-28.

23. Chang L, Graham PH, Ni J, et al. Targeting PI3K/Akt/ mTOR signaling pathway in the treatment of prostate cancer radioresistance. Crit Rev Oncol Hematol 2015; 96: 507-17.
24. Hu K, Dai HB, Qiu ZL. mTOR signaling in osteosarcoma: oncogenesis and therapeutic aspects (review). Oncol Rep 2016; 36: 1219-25.

25. King D, Yeomanson D, Bryant HE. PI3King the lock: targeting the $\mathrm{PI} 3 \mathrm{~K} / \mathrm{Akt} / \mathrm{mTOR}$ pathway as a novel therapeutic strategy in neuroblastoma. J Pediatr Hematol Oncol 2015; 37: 245-51.

26. Zhao JJ, Chen PJ, Duan RQ, Li KJ, Wang YZ, Li Y. miR-630 functions as a tumor oncogene in renal cell carcinoma. Arch Med Sci 2016; 12: 473-8.

27. Zhang C, Li S, Qiao B, et al. CtBP2 overexpression is associated with tumorigenesis and poor clinical outcome of prostate cancer. Arch Med Sci 2015; 11: 1318-23. 